\title{
Kidney and heavy metals - The role of environmental exposure (Review)
}

\author{
PAOLO LENTINI $^{1 *}$, LUCA ZANOLI $^{2 *}$, ANTONIO GRANATA $^{3}$, SALVATORE SANTO SIGNORELLI $^{2}$, \\ PIETRO CASTELLINO ${ }^{2}$ and ROBERTO DELL'AQUILA ${ }^{1}$ \\ ${ }^{1}$ Department of Nephrology and Dialysis, ‘St. Bassiano’ Hospital, I-36061 Bassano del Grappa; \\ ${ }^{2}$ Department of Internal Medicine, University of Catania, I-95124 Catania; ${ }^{3}$ Department \\ of Nephrology ‘St. Giovanni di Dio’ Hospital, I-92100 Agrigento, Italy
}

Received January 2, 2017; Accepted February 24, 2017

DOI: $10.3892 / \mathrm{mmr} .2017 .6389$

\begin{abstract}
Heavy metals are extensively used in agriculture and industrial applications such as production of pesticides, batteries, alloys, and textile dyes. Prolonged, intensive or excessive exposure can induce related systemic disorders. Kidney is a target organ in heavy metal toxicity for its capacity to filter, reabsorb and concentrate divalent ions. The extent and the expression of renal damage depends on the species of metals, the dose, and the time of exposure. Almost always acute kidney impairment differs from chronic renal failure in its mechanism and in the magnitude of the outcomes. As a result, clinical features and treatment algorithm are also different. Heavy metals in plasma exist in an ionized form, that is toxic and leads to acute toxicity and a bound, inert form when metal is conjugated with metallothionein and are then delivered to the liver and possible causing the kidney chronic damage. Treatment regimens include chelation therapy, supportive care, decontamination procedures and renal replacement therapies. This review adds specific considerations to kidney impairment due to the most common heavy metal exposures and its treatment.
\end{abstract}

\section{Contents}

1. Introduction

Correspondence to: Dr Paolo Lentini, Department of Nephrology and Dialysis, ‘St. Bassiano’ Hospital, I-36061 Bassano del Grappa, Italy

E-mail: paolo.lentini@yahoo.it

*Contributed equally

Abbreviations: AKI, acute kidney injury; CKD, chronic kidney disease; RRTs, renal replacement therapies; HD, hemodialysis; PD, peritoneal dialysis; CRRTs, continuous renal replacement therapies; CVVH, continuous venous-venous hemofiltration; CVVHDF, continuous venous-venous hemodiafiltration; HMP, hemoperfusion; TPE, therapeutic plasma exchange

Key words: arsenic, cadmium, kidney, iron, lead, mercury, pollution, toxicity
2. Mechanism of heavy metal toxicity

3. Symptoms of exposure and toxicity

4. Laboratory testing

5. Ultrasound examination

6. Treatment

7. Lithium

8. Cisplatin

9. Cadmium

10. Arsenic

11. Mecrcury

12. Lead

13. Other heavy metals

14. Conclusion

\section{Introduction}

Heavy metals are chemical elements with a specific gravity, a measure of density compared to an equal amount of water, 5 times higher than the specific gravity of water $\left(1-4 C^{\circ}\right)$. Availability of heavy metals is determined by physical factors (phase association, temperature, adsorption), chemical factors (lipid solubility, kinetics) and biochemical factors (human trophism) (1).

Therapeutic, accidental or occupational exposure to several metals may cause renal toxicity. The most common metals implicated in kidney toxicity are arsenic, barium, cadmium, cobalt, copper, lead, lithium, mercury and platinum. Small amounts of these trace elements are necessary for good health because they are important factors and cofactors in many biochemical cellular pathways, including nucleic acids and proteins synthesis, and enzymatic reactions.

A large amount of heavy metals results from agriculture and industrial activities such as production of pesticides, batteries, alloys, and textile dyes but natural phenomena such as earthquakes, volcanic eruptions have also been reported to significantly contribute to environmental pollution (2).

\section{Mechanism of heavy metals toxicity}

Heavy metals may enter chronically into the human body through food, water, air or skin whereas acute poisoning is 
the result from inhalation or skin contact of vapors, fumes, dust in workplace, or an inappropriate use of some therapeutic measures. Accidental ingestion is the most common route of exposure in children. Heavy metal toxicity results in damage of the central nervous system, blood, lungs, kidneys, liver and other organs; diagnostic medical devices, such as the use of barium or the direct injection of gallium during radiological examination, are also unexpected forms of heavy metal toxicity.

The kidney is a target organ in heavy metal toxicity for its capability to reabsorb and concentrate divalent ions and metals. The magnitude of kidney impairment depends on the nature, the dose, and the time of exposure.

Acute kidney injury (AKI) by heavy metals diverge from chronic kidney disease (CKD) for the mechanism of toxicity. For this reason, clinical features and a therapeutic approach are also peculiar (3).

Heavy metals in plasma exist in two different patterns: protein bound (non-diffusible) and complex/ionized (diffusible) forms. Metals are quickly cleared from the blood and are sequestered in many tissues.

The luminal fluid in the early proximal tubule can contain both the bound form and the free form. In chronic intoxication the protein bound, inert form is conjugated with metallothionein and glutathione, which are then released into the blood by the liver and the kidney. These compounds are subsequently reabsorbed through an endocytotic process in segment S1 of the proximal tubule and can lead to chronic inflammation, fibrosis and renal failure $(3,4)$. In acute intoxication, the main site of reabsorption is the apical membrane of the first zone of the proximal tubule, but also the loop of Henle and the terminal segments can participate in reabsorption of heavy metals. AKI is induced by the ionized form that produces direct cellular toxicity, cellular membrane disruption and uncoupling of the mitochondrial respiration pathway, with the release of numerous apoptosis signals such as cytokines and reactive oxygen species (4).

\section{Symptoms of exposure and toxicity}

Exposure to toxic heavy metals is generally classified as acute (1-14 days), intermediate (15-354 days) and chronic ( $\geq 365$ days). Acute toxicity is abrupt or accidental contact or ingestion of high dose of heavy metals. Symptoms of acute toxicity are severe, rapid in onset and associated with exposure or ingestion, headache, vomiting, dyspnoea, abdominal pain and sweating, whereas chronic intoxication is usually uneventful. Medical history and specific tests can help to diagnose the specific agent.

\section{Laboratory testing}

The diagnosis of heavy metal toxicity requires observation of presenting symptoms, the history of potential exposure and the result of laboratory tests. Specific tests include blood count, liver and renal function, urinalysis, faecal tests, X-rays and fingernail analysis. Given the limitations of serum creatinine as a marker of renal function, different urinary and serum molecules have been investigated as possible markers of acute or chronic kidney diseases. One of the most promising novel biomarkers is neutrophil gelatinase-associated lipocalin (NGAL) (5). Urinary NGAL has also been shown to predict kidney injury and dialysis requirement in welding workers (6). Pennemans et al (7) showed that urinary kidney injury molecule-1 levels are positively correlated with urinary cadmium concentration. Biomarkers and nephrotoxicity (e.g., renal tubular injury) will be an emerging concern in occupational health.

\section{Ultrasound examination}

Despite not yet coded for kidney damage secondary to heavy metal exposure, renal ultrasound examination may potentially have a role in the differentiation of acute and chronic damage $(8,9)$.

\section{Treatment}

Treatment regimens vary significantly and are tailored to each specific individual's medical condition and the circumstance of their exposure; a complete history of possible ingestion, occupation, recreational activities and environment is critical in the diagnosis of heavy metal toxicity.

Therapeutic approaches include supportive and decontamination procedures (such as charcoal, cathartics, emesis, gastric lavage), supportive care (e.g., intravenous fluids, cardiac stabilization, mechanical ventilation, exchange transfusion), chelation therapy, and renal replacement therapies (RRTs); if hemodynamic instability occurs, continuous renal replacement therapies (CCRTs), especially continuous venous-venous hemofiltration $(\mathrm{CVVH})$ and continuous venous-venous hemodiafiltration (CVVHDF) are the selected extracorporeal regimens. Hemodialysis (HD) and peritoneal dialysis (PD) are often used, especially in acute intoxication without relevant hypotension, impaired mental status or dyspnoea. The clinical and therapeutic algorithm should take into account age, cardiovascular disease, liver and kidney disease that can reduce endogenous clearance; toxicological parameters such as apparent distribution volume, protein binding, molecular weight, elimination half-life, toxic and lethal dose (10). The kidney impairment and the therapeutic approaches to the most common toxic metals, previously analysed by our group (9), is reported in Table I.

\section{Lithium}

Lithium (Li) carbonate is a common medication prescribed for the treatment of bipolar manic-depressive disorder. Acute and chronic maintenance therapy with lithium may lead to severe toxicity.

Sources of exposure. Lithium ingestion can occur accidentally or in suicide attempts. Anti-depressive therapy may also lead to excessive exposure.

Mechanism of kidney damage. Lithium is a small ion that does not bind to proteins; however, lithium diffusion between intracellular and extracellular compartments is slow. Lithium carbonate is almost totally absorbed by the tissues within $8 \mathrm{~h}$ after distribution into the intravascular space. Therefore, there 
Table I. Therapeutic approaches in acute heavy metal toxicity [adapted from Lentini et al (11)].

\begin{tabular}{|c|c|c|c|c|c|c|c|}
\hline Metal & HD & PD & $\mathrm{CVVH}$ & CVVHDF & TPE & HMP & Chelators \\
\hline Arsenic & $\begin{array}{l}\text { HD+DMSA } \\
\text { or HD+BAL }\end{array}$ & $\begin{array}{l}\text { PD+DMSA } \\
\text { or PD+BAL }\end{array}$ & Unknown & Unknown & Unknown & Unknown & $\begin{array}{l}\text { BAL, DMSA, } \\
\text { D-penicillamine }\end{array}$ \\
\hline Cadmium & No & No & No & No & No & No & Calcium-EDTA \\
\hline Copper & $\begin{array}{c}\mathrm{HD}+ \\
\text { D-penicillamine }\end{array}$ & Unknown & Unknown & $\begin{array}{c}\text { CVVHDF+ } \\
\text { D-penicillamine }\end{array}$ & No & Yes & $\begin{array}{c}\text { D-penicillamine } \\
\text { BAL }\end{array}$ \\
\hline Lead & No & No & No & No & Unknown & No & $\begin{array}{c}\text { Calcium-sodium } \\
\text { EDTA, BAL, } \\
\text { DMSA }\end{array}$ \\
\hline Lithium & Yes & Yes & Yes & Yes & No & No & No \\
\hline Mercury & No & No & No & CVVHDF+DMPS & Unknown & No & $\begin{array}{c}\text { BAL, DMSA, } \\
\text { DMPS } \\
\text { (inorganic only) }\end{array}$ \\
\hline Platin & Yes & Yes & Unknown & Unknown & Yes & No & No \\
\hline
\end{tabular}

HD, hemodialysis; PD, peritoneal dialysis; CVVH, continuous venous-venous hemofiltration; CVVHDF, continuous venous-venous hemodiafiltration; TPE, therapeutic plasma exchange; HMP, hemoperfusion; DMSA, dimercaptosuccinic acid; BAL, dimercaprol; EDTA, ethylenediaminetetraacetic acid; DMPS, dimercapto-1-propane sulfonate.

is a quick disappearance from the plasma and a slow excretory phase, and $95 \%$ of a dose is excreted in urine; $30-60 \%$ during the first $12 \mathrm{~h}$ and the remaining part during the next 10-14 days (12).

Clinical and laboratory features. Patients with acute lithium intoxication can present confusion, hyperreflexia, tremor, stupor, diarrhoea, vomiting and hemodynamic instability with or without AKI. Renal presentation may occur with polyuria and casts in the sediment. Chronic lithium ingestion is a common cause of nephrotic syndrome (to minimal change or with focal-segmental glomerulosclerosis pattern with collapsed glomeruli), chronic interstitial nephropathy renal tubular acidosis or nephrogenic diabetes insipidus (13).

Laboratory tests. Serum lithium therapeutic range is 0.4-1.2 mmol/1. Values exceeding this range are highly suggestive of intoxication. However, values in the normal range do not exclude toxic exposure.

\section{Treatment of acute lithium intoxication}

Supportive measures. Patients with normal renal function should initially be treated with a rapid infusion of saline with sodium bicarbonate to increase the urinary lithium output. Emetics administration with gastric lavage should be delivered within $8 \mathrm{~h}$ after acute overdose. If the patients present a severe intoxication with AKI, coma, convulsions and respiratory failure, a cardiac monitoring, mechanical ventilation and RRTs are recommended.

Extracorporeal therapies. To accelerate lithium clearance in presence of levels $>3.5 \mathrm{mmol} / 1$, as in the case of acute intoxication, various modalities of extracorporeal blood purification may provide adequate results. Lithium is rapidly removed by HD diffusion for its structure of small, non-protein bound molecule with simultaneous correction of acid-base and electrolyte disorders. The efficiency of extracorporeal removal is limited by a high postdialysis rebound of lithium levels due to compartmentalization of the molecule. High-efficiency HD is recommended. Mixed diffusive-convective therapies, such as hemodiafiltration (HDF) and dialysis with high-flux dialyzer [high flux dialysis paired with membrane with polysulfone, polyamide, polymethylmethacrylate (PMMA)], seem to be even more efficient, and represent the first-choice therapy for severe acute lithium intoxication (10). Lithium can be removed also by PD, but the clearance is lower than obtained with HD, HDF and high flux dialysis. Therefore, PD can be considered a valid option only when HD is not available (14). Because the adsorption of lithium with activated charcoal or resins is very limited, and the technique of hemoperfusion (HMP) is poorly efficient. CVVH and CVVHDF can result in a conspicuous blood depuration for the continual removing of intracellular lithium and the low postdialysis rebound. In cases of acute lithium poisoning, continuous renal replacement therapy (CRRT) does not decrease lithium level as rapidly as HD does. Therefore, the best treatment for acute lithium intoxication appears to be the combination of $\mathrm{HD}$, for rapid lithium removal, followed by CVVHDF or CVVH, to prevent postdialysis rebounding (14).

\section{Cisplatin}

Cisplatin is a common chemotherapic agent used against various types of solid and haematological malignancies; common side effects of cisplatin include myelotoxicity, neurotoxicity, ototoxicity and nephrotoxicity.

Sources of exposure. The typical source of exposure is chemotherapy.

Mechanism ofkidney damage. Cisplatin toxicity is dose-related. It is a strong renal tubular toxin that can injure the S3 segment 
cells of the proximal tubule with a variable involvement of the distal nephron. The earliest change in proximal tubule function is the rapid production, via cytochrome P450 enzymes, of highly reactive hydroxyl radicals that produce injury by DNA binding that lead to decreased protein synthesis (15).

Clinical and laboratory features. Accidental or suicidal attempt overdose of cisplatin may result in nausea, constipation, hearing loss, vomiting or gross haematuria, and reduce urine output.

Laboratory tests. Liver failure, expressed by elevated bilirubin values, alanine transferase (ALT) aspartate transferase (AST), $\gamma$-glutamyl transferase (GGT), and elevated markers of renal dysfunction are common findings during acute cisplatin intoxication. Cisplatin is commonly associated with anaemia for the erythropoietin deficiency induced by kidney injury. Macroscopic or microscopic haematuria with urine casts are common. The urine osmolarity is similar to that of plasma; this concentrating defect reflects platinum-induced damage of the loop of Henle.

\section{Treatment of acute cisplatin intoxication}

Chelating agents. There is no specific chelation therapy for cisplatin intoxication. An organic thiophosphate, i.e., amifostine, may attenuate cisplatin-induced toxicity by donating a protective thiol group and reducing oxidative stress.

Supportive measures. Hydration with electrolyte solutions, forced diuresis and antiemetic therapies represent the most common measures to prevent cisplatin-induced nephrotoxicity; myelosuppression frequently requires the administration of granulocyte colony-stimulating factors (16).

Extracorporeal therapies. HD is able to reduce free cisplatin in plasma. However, after administration, the metal binds to plasma proteins very quickly and cannot be further eliminated by this procedure. Moreover, there is also a large rebound of cisplatin into plasma from an exchangeable pool after HD. Therapeutic plasma exchange (TPE) appears able to remove both the protein-bound fraction and the cisplatin free form. Hofman et al used a plasma filter and the substitution of 3 liters of plasma for three to four sessions (16).

\section{Cadmium}

Cadmium (Cd) can induce critical toxicity in humans. There are many cases of chronic intoxication after cadmium exposure but only a few cases of acute poisoning from oral ingestion or accidental inhalation of cadmium-containing fumes.

Sources of exposure. Cadmium is present in major industrial and chemical applications, such as in the production of alloys and batteries. Operation involving removal of cadmium paints by scraping or blasting may pose a significant hazard in shipyard employment, construction industry, and the agricultural industry. Exposure to cadmium mainly results from working in cadmium contaminated work places and, less commonly, in eating contaminated food or smoking cigarettes.

\section{Mechanism of kidney damage}

Acute exposure. The ionized free form induces cellular toxicity reducing phosphate and glucose transport and inhibiting mitochondrial respiration, with membrane rupture of the proximal tubular cells of the nephron (17).

Chronic exposure. After ingestion or inhalation, cadmium is transported to the liver and to the kidney by metallothionein, which binds cadmium. Signs of cell apoptosis and cytokine pathway activation are common in this syndrome. A typical, chronic tubular-interstitial nephropathy is produced by the accumulation of this metal in the medulla and S1 segment of the proximal tubule.

\section{Clinical and laboratory features}

Acute exposure. The toxic symptoms include mental status alteration, vomiting, nausea and dyspnoea, with hypotension, shock, and acute renal and liver failure.

Chronic exposure. CKD, emphysema, cough and gastrointestinal bleeding results during chronic exposure. Cadmium-related renal impairment occurs with polyuria, loss of concentration capacity, tubular proteinuria, renal glycosuria, aminoaciduria, hyperphosphaturia and hypercalciuria.

Laboratory tests. Exposure to cadmium is commonly determined by measuring the 24-h urinary cadmium excretion; an elevated urinary excretion of $\beta 2$-microglobulin has proved to be useful in detecting the more subtle signs of cadmium nephrotoxicity (18).

\section{Treatment of acute cadmium exposure}

Chelating agents. There are no antidotes for cadmium intoxication: almost immediately after absorption, cadmium is bound with metallothionein and stored in the erythrocytes. In contrast to the other heavy metals, chelators seem to increase cadmium nephrotoxicity (18).

Supportive measures. Gastrointestinal decontamination must be performed within few hours from ingestion with support for cardiac and pulmonary function; forced diuresis is not indicated due to cadmium high nephrotoxicity.

Extracorporeal therapies. The extracorporeal measures of detoxification are ineffective, because cadmium is fixed to cells; PD, HD, and CRRT are used to remove chelators in acute renal failure caused by cadmium $(11,12)$.

\section{Arsenic}

Arsenic (As) exists in organic forms (the trivalent and pentavalent forms) and in inorganic forms (arsine gas, arsenite, and arsenate). Acute high-dose intoxication may cause death and severe systemic toxicity.

Source of exposure. The most toxic forms are the trivalent arsenic or arsenite compounds. Common sources of exposure are herbicides, pesticides, contaminated water, food supplies and homeopathic remedies.

Mechanism of kidney damage. Arsenic compounds are fully absorbed after ingestion or inhalation. On entering the circulation, arsenic strictly binds haemoglobin. After $24 \mathrm{~h}$, it is accumulated in soft tissue; after 2 weeks, arsenic is incorporated in hair and nails.

The most toxic form, the trivalent arsenic, intensely binds to sulfhydryl groups and inhibit enzyme systems such as those 
of cellular respiration, with uncoupling oxidative phosphorylation (19).

\section{Clinical and laboratory features}

Acute exposure. Acute exposure can arise after acute inhalation in high levels of arsenic dusts or fumes, or ingestion in suicide or poisoning attempts. Acute toxicity symptoms include headache, vomiting, nausea, abdominal pain and diarrhoea, frequently followed by macula rash, diffuse itch, dehydration, respiratory failure and acute respiratory distress syndrome, hypotension and hemodynamic instability. AKI may result in acute tubular necrosis, haemoglobinuria due to haemolysis, haematuria, oliguria and proteinuria (19).

Chronic exposure. In chronic poisoning, peripheral neuropathy and encephalopathy with cognitive impairment are the predominant manifestations.

Laboratory tests. In acute exposure, urine arsenic levels are more relevant than hematic measurements because arsenic is rapidly cleared from the blood. Excretion of more than $200 \mu \mathrm{g}$ in a $24 \mathrm{~h}$ urine collection is suggestive of arsenic overload. Chronic arsenic exposure can be confirmed by a $24 \mathrm{~h}$ urine collection and arsenic concentration determination. Hair and nails can confirm the diagnosis.

\section{Treatment of acute arsenic intoxication}

Chelating agents. Chelating agents represent a key treatment in a severely ill patients with acute arsenic poisoning, BAL and dimercapo-1-propane sulfonate are the most frequently used agents.

Supportive measures. The first step is the elimination of further exposure. Gastrointestinal decontamination with charcoal and forced emesis is recommended. Furthermore, because arsenic is well eliminated by urine, it is useful to force diuresis. In severe arsenic intoxication, exchange transfusion has been largely used with some benefit.

Extracorporeal therapies. HD can be used to remove chelators that are nephrotoxic but it has very limited efficiency in arsenic removal; moreover HD and other extracorporeal blood purification techniques should be used if AKI develops. CVVH-CVVHDF are preferred to carry on hemodynamic stability (12). PD and HMP are inefficient and are not indicated (12).

\section{Mercury}

Mercury $(\mathrm{Hg})$ is a silvery white liquid that is volatile at room temperature because of its high vapour pressure. Mercury exists in three forms: elemental, inorganic and organic.

Source of exposure. The general population is primarily exposed to the organic forms by fish (tuna, swordfish) and inorganic form by amalgam fillings. Occupational exposure occurs in alloys and thermometer factories, chloralkali industries and in dentistry (20).

Mechanism of kidney damage. After acute exposure to mercury, acute tubular necrosis appears, usually accompanied by oligo-anuria. In chronic exposure mercury is stored in the kidney and induces epithelial injury and necrosis in the pars recta of the proximal tubule (21).
Clinical and laboratory features

Acute exposure. Elemental mercury in vapours produces acute dyspnoea, abdominal pain, altered mental status, profuse salivation chills, vomiting, tremors and hypotension.

Chronic exposure. Organic mercury gives skin manifestations and neurological disturbances such as hearing loss, paraesthesia and ataxia. Mercury-related kidney damage can due to tubular dysfunction with elevated urinary excretion of albumin, transferrin, retinol binding protein, and $\beta$-galactosidase and a nephrotic syndrome with membranous nephropathy pattern (21).

Laboratory tests. A mercury concentration $>45 \mathrm{mg} / \mathrm{dl}$ in blood indicate acute poisoning.

\section{Treatment of acute mercury exposure}

Chelating agents. The antidotes currently available are BAL, DMPS, and dimercaptosuccinic acid (DMSA). Treatment with chelators should be established especially in patients with acute symptoms arising from the central nervous system.

Supportive measures. A quick elimination of mercury is necessary and can be obtained by gastrointestinal decontamination and rapid administration of chelators, followed by intensive monitoring of hemodynamics and breathing.

Extracorporeal therapies. Plasma protein binding of mercury is $95 \%$, and the toxin is distributed in a large apparent volume of distribution; for these reasons, HD, PD and HMP with charcoal are poorly efficient. On the other hand, HD is useful to eliminate chelators that are highly water-soluble whereas CVVH is more effective in removing the complex mercury-DMPS (22). Furthermore, TPE is the most efficient treatment to remove inorganic mercury and could be useful in association with chelation therapy (20).

\section{Lead}

Lead $(\mathrm{Pb})$ is the most pervasive of the nephrotoxic metals, and humans are exposed to this agent in water, food and air.

Source of exposure. Lead exists in three different forms: organic form, such as tetramethyl lead (which is the most toxic form), metallic lead and inorganic lead (water-soluble lead salts).

Acute exposure. Acute intoxication is extremely rare and occurs after accidental inhalation of tetramethyl or accidental or suicidal ingestion of water-soluble inorganic lead salts.

Chronic exposure. Lead-glazed ceramics, lead paint, and herbal remedies from Asia are potential sources of lead exposure. Workers in manufacture of ammunition, batteries, sheet lead, bronze plumbing, radiation shields, and intravenous pumps have a high exposure. Lead also contaminates emissions from motor cars with antiknock additives (tetramethyl lead) (23).

\section{Mechanism of kidney damage}

Acute exposure. Acute lead poisoning causes impairment of the proximal tubular architecture and histological changes, such as eosinophilic intranuclear inclusions in tubular cells consisting of lead-protein complexes and mitochondrial swelling (22). 
Chronic exposure. The damage may be revealed by the increased urate secretion, vasoconstriction, and consequent glomerulosclerosis, hypertension and interstitial fibrosis $(23,24)$.

\section{Clinical and laboratory features}

Acute exposure. Severe toxicity, with a blood lead level of $50 \mu \mathrm{g} / \mathrm{dl}$ or more, affects the central and peripheral nervous systems, with frank paralysis, tremors, decreased nerve conduction velocity and papilledema.

Lead poisoning produces also an altered, characteristic metallic taste, abdominal pain with vomiting and nausea, muscle weakness and acute haemolytic anaemia. Renal impairment may reveal acute tubular necrosis with microscopic haematuria, casts, and aminoaciduria with oliguric AKI. This damage occurs in 1-2 days after exposure.

Chronic exposure. Patients present with myalgia, fatigue, dyspnoea, non-specific abdominal pain, and anorexia. Renal damage involves tubular disorders with Fanconi-like syndrome, glycosuria, aminoaciduria and phosphaturia.

Laboratory tests. The lead level in the whole blood is an indicator of recent exposure; the selected diagnosis to evaluate the lead level is the ethylenediaminetetraacetic acid (EDTA) lead mobilization test (22). Elevation of blood urea nitrogen, creatinine, and serum uric acid with urinary amino acids, glucose, and ALA (proximal tubule damage) are common laboratory features as well as elevated reticulocyte count and normo-hypochromic anaemia.

\section{Treatment of acute lead intoxication}

Chelating agents. In an inorganic lead intoxication, there is an indication to use EDTA, BAL, DMSA and D-penicillamine.

Supportive measures. Gastric lavage and decontamination with activated charcoal are indicated if lead salts have been ingested. Fluid-electrolyte balance must be maintained. Diuretic therapy is indicated, not to eliminate lead but to remove the chelators (12).

Extracorporeal therapies. Extracorporeal detoxification measures are ineffective because $95 \%$ of lead is stored in the erythrocytes. However, chelators, which are nephrotoxic, can be effectively removed by HD. The half-life of lead in blood is $9 \mathrm{~h}$ during combined HD and EDTA and $96 \mathrm{~h}$ when EDTA is given alone. PD, HMP, CRRT and TPE are generally ineffective $(12,25)$.

\section{Other heavy metals}

Medical literature reports few cases of acute renal failure caused by other heavy metals such as copper, aluminium, vanadium, uranium, bismuth salts and hexavalent chromium. Evidence for treatment for poisoning by these agents is narrow and not well coded (26).

\section{Conclusion}

Acute heavy metal toxicity must be distinct from chronic toxicity in its management. With the improvements in industrial safety, cases of acute heavy metal poisoning are uncommon but they remain a cause of AKI that should always be considered, especially in presence of accidental contamination, environmental or industrial catastrophes or suicide attempts. Chelation therapy is the preferred medical treatment to reduce the toxic effects of heavy metals. In this regard, RRTs are essential and crucial to accelerate detoxification and to improve the outcome of patients with acute poisoning. These treatments play also an important role in renal detoxification related to nephrotoxicity of chelating agents. The use of CRRT, particularly CVVH and HDF, as yet not well codified, appears to offer advantages in presence of hemodynamic instability without reducing the purifying effect.

\section{References}

1. Freundlich HMFL Characteristics of heavy metals. J Phy Chem 57: 385-470, 1906 (In German).

2. Beliles RP: The lesser metals, In: Toxicity of Heavy Metals in the Environment, Part 2. Oehme FW (ed). Marcel Dekker, New York, NY, p383, 1979.

3. Sabolić I: Common mechanisms in nephropathy induced by toxic metals. Nephron, Physiol 104: 107-114, 2006

4. Barbier O, Jacquillet G, Tauc M, Cougnon M and Poujeol P: Effects of heavy metals on and handling by the kidney. Nephron, Physiol 99: 105-110, 2005.

5. Lentini P, de Cal M, Clementi A, D'Angelo A and Ronco C: Sepsis and AKI in ICU patients: The role of plasma biomarkers. Crit Care Res Pract 2012: 856401, 2012.

6. Tchounwou PB, Yedjou CG, Patlolla AK and Sutton DJ: Heavy metals toxicity and the environment. In: Molecular, Clinical and Environmental Toxicology. Luch A (ed). Vol. 101. Springer Basel, New York, NY, pp133-164, 2012.

7. Pennemans V, De Winter LM, Munters E, Nawrot TS, Van Kerkhove E, Rigo JM, Reynders C, Dewitte H, Carleer R, Penders $\mathrm{J}$, et al: The association between urinary kidney injury molecule 1 and urinary cadmium in elderly during long-term, low-dose cadmium exposure: A pilot study. Environ Health 10: 77, 2011.

8. Granata A, Zanoli L, Clementi S, Fatuzzo P, Di Nicolò P and Fiorini F: Resistive intrarenal index: Myth or reality? Br J Radiol 87: 20140004, 2014.

9. Granata A, Zanoli L, Insalaco M, Valentino M, Pavlica P, Di Nicolò PP, Scuderi M, Fiorini F, Fatuzzo P and Bertolotto M: Contrast-enhanced ultrasound (CEUS) in nephrology: Has the time come for its widespread use? Clin Exp Nephrol 19: 606-615, 2015.

10. Locatelli C: Italian Manual of Dialysis. Witching, Milano, pp533-552, 1999 (In Italian).

11. Lentini P, Poghosyan L and Ronco C: Lead, Heavy Metals and the Kidney. In: Critical Care Nephrology. 2nd edition. Saunders, Philadelphia, pp1705-1711, 2009.

12. Seyfart G: Poison Index: Heavy Metals Intoxication. 4th edition. Pabst Science Publishers, Berlin, pp390-410, 1997.

13. Aurell M, Svalander C, Wallin L and Alling C: Renal function and biopsy findings in patients on long-term lithium treatment. Kidney Int 20: 663-670, 1981.

14. Peces R, Fernández EJ, Regidor D, Peces C, Sánchez R, Montero A and Selgas R: Treatment of acute lithium intoxication with high-flux haemodialysis membranes. Nefrologia 26: 372-378, 2006 (In Spanish).

15. Basnakian AG, Apostolov EO, Yin X, Napirei M, Mannherz HG and Shah SV: Cisplatin nephrotoxicity is mediated by deoxyribonuclease I. J Am Soc Nephrol 16: 697-702, 2005.

16. Hofmann G, Bauernhofer T, Krippl P, Lang-Loidolt D, Horn S, Goessler W, Schippinger W, Ploner F, Stoeger H and Samonigg H: Plasmapheresis reverses all side-effects of a cisplatin overdose - A case report and treatment recommendation. BMC Cancer 6: 1-7, 2006.

17. Fowler BA: Mechanisms of kidney cell injury from metals. Environ Health Perspect 100: 57-63, 1993.

18. Wu X, Jin T, Wang Z, Ye T, Kong Q and Nordberg G: Urinary calcium as a biomarker of renal dysfunction in a general population exposed to cadmium. J Occup Environ Med 43: 898-904, 2001.

19. Aposhian HV, Maiorino RM and Dart DC: Biochemical toxicology of arsenic. Rev Biochem Toxicol 10: 265-271, 1989. 
20. Clarkson TW, Magos L and Myers GJ: The toxicology of mercury - Current exposures and clinical manifestations. N Engl J Med 349: 1731-1737, 2003.

21. Zalups RK: Molecular interactions with mercury in the kidney. Pharmacol Rev 52: 113-143, 2000.

22. Pai P, Thomas S, Hoenich N, Roberts R, House I and Brown A: Treatment of a case of severe mercuric salt overdose with DMPS (dimercapo-1-propane sulphonate) and continuous haemofiltration. Nephrol Dial Transplant 15: 1889-1890, 2000.

23. Patrick L: Lead toxicity, a review of the literature. Part 1: Exposure, evaluation, and treatment. Altern Med Rev 11: 2-22, 2006.
24. Loghman-Adham M: Renal effects of environmental and occupational lead exposure. Environ Health Perspect 105: 928-938, 1997.

25. Pedersen RS: Lead poisoning treated with haemodialysis. Scand J Urol Nephrol 12: 189-190, 1978.

26. Kiss JE, Berman D and Van Thiel D: Effective removal of copper by plasma exchange in fulminant Wilson's disease. Transfusion 38: 327-331, 1998. 